\title{
Identification of Terminal Connection and System Function for Sensitive Frequency Response Measurement on Transformers
}

\author{
L. Satish, Senior Member, IEEE, and A. Saravanakumar, Student Member, IEEE
}

\begin{abstract}
Frequency response measurement on a transformer is a low voltage, off-line exercise. So, it virtually permits determination of any network/system function, by imposing any desired terminal condition for the nontested windings. The terminal conditions employed have significant influence on the achievable fault detection ability, and maximizing this ability should obviously be one of the main aims of frequency response measurements. Simply stated, this requirement translates to the ability to identify/measure as many natural frequencies as possible. However, there is a practical limitation that not all network/system functions can exhibit all natural frequencies. Hence, it is necessary to determine the most appropriate combination of terminal connection and system function for achieving this objective. Considering a two-winding transformer representation, system functions for different terminal conditions were computed. Depending on number of natural frequencies distinguishable in the amplitude frequency response of a system function, each measuring condition was ranked. Thus, it led to identification of the best configuration. Later, these findings were verified on an actual transformer. Performance of best configuration was compared with currently employed low-voltage impulse (LVI) test (used during short-circuit testing of transformers) and sweep frequency response measurement test conditions, and found to be better. In conclusion, it is believed that after adequate field verifications, the identified configuration can be declared as the preferred way of making frequency response measurements on transformers.
\end{abstract}

Index Terms-Natural frequencies, nontested windings, sensitivity, sweep frequency response, terminal connections, transformers.

\section{INTRODUCTION}

D URING high-voltage (HV) impulse tests on transformers, it is mandatory to short-circuit and ground (directly or via a resistance) all nontested windings to prevent their accidental damage due to transferred/induced voltages, which may, at times, exceed the BIL limit [1], [2]. The guidelines concerning terminal connection of nontested windings were formulated primarily to ensure safety of windings (rather than sensitivity considerations).

In recent years, sweep frequency response measurement (popularly referred to as FRA, SFRA, etc.) is increasingly being

Manuscript received December 6, 2006; revised July 19, 2007. Paper no. TPWRD-00775-2006.

The authors are with the High Voltage Laboratory, Department of Electrical Engineering, Indian Institute of Science, Bangalore, India (e-mail: satish@hve. iisc.ernet.in).

Digital Object Identifier 10.1109/TPWRD.2008.915808 used to assess mechanical integrity of transformer windings. These being off-line, low voltage measurements, precautions similar to those in a HV test are obviously not necessitated. Moreover, the primary objective in these low-voltage tests (in contrast to HV tests) is to achieve the highest possible fault-detection ability. So, it has to be determined whether terminal conditions currently being employed satisfies this requirement. Moreover, there are no universally agreed upon procedures for making frequency response measurements. But, literature reveals that during the early days of FRA measurement, the practice was to keep nontested windings open-circuited and floating [3]-[5]. However, in recent years due to the advent of powerful and customized frequency response measuring instruments (specifically meant for transformers), the practice has undergone a change. It emerges that nontested windings are kept open and/or short circuited with neutral floating (and employing a $50 \Omega$ termination during measurements) is the popularly followed practice by the industry and power utilities [6]. Perhaps, due to experience gained, this practice has emerged as a de facto standard. However, it is appropriate to mention that there is no theoretical background to demonstrate that this choice is indeed the best. Because of this, it remains to be verified whether by adopting this practice, the possibility of achieving a higher level of sensitivity (obtainable from another connection) has not been precluded. Therefore, this study gathers significance and is aimed at identification of the most favorable test configuration for making sensitive frequency response measurements. It is needless to emphasize the implications of this investigation.

Incidentally, low-voltage impulse (LVI) test is an accepted off-line method for detecting winding deformation during short-circuit testing of transformers [7], [8]. The relevant standards specify two alternatives for terminal connections, of which, the difference-current measurement is indicated as preferred (quoting sensitivity reasons). In summary, it emerges that nontested windings have been connected in a variety of ways, viz.

- HV impulse test: short-circuited and grounded;

- LV impulse test: Line-end open-circuited and neutral-end grounded;

- SFRA test:

1) Open-circuited and floating (Earlier practice)

2) Open and/or short circuited and neutral floating, measured with $50 \Omega$ termination (Current practice)

Therefore, it becomes essential to determine what would be the ideal terminal condition and pertinent system function, for 
achieving the maximum sensitivity during frequency response measurements. This investigation is expected to provide the necessary answers.

\section{OBJeCtIVES}

The natural frequencies (i.e. poles) of a transformer is dependent on several factors like, type of winding, their numbers, spatial position and arrangement, clearances, terminal configurations, as well as, interaction with neighboring windings. So, there exists a correlation between natural frequencies and mechanical status of winding assembly. Thus, intuitively speaking, knowledge about all natural frequencies would indicate the highest degree of sensitivity achievable in detecting winding deformation. In other words, for higher sensitivity, it is vital to have access to all the natural frequencies. If for any reason, information about some of them is unavailable, then the measurement is less sensitive to that extent.

Amongst the factors that influence natural frequencies of a transformer, terminal configuration is the only one that can be externally manipulated, while the remaining ones are not. Also, depending on the measured quantity, some natural frequencies may be nonexcitable, either because they lie close to a zero, or they are close to a dominant pole, or damping is high. A good example of this is the neutral current in an interleaved-disc winding, wherein, all high-frequency poles lie very close to zero and hence are almost cancelled out, and therefore nonexcitable [9]. Whereas, the same natural frequencies are observable when disc-to-disc voltages are measured [10]. Hence, the quantity being measured is important. With these objectives in background, this contribution seeks to answer the following question (initially by considering a two-winding transformer).

- During sweep frequency response measurements, what combination of terminal connection and system function (viz. transfer or driving-point) would be most appropriate to adopt?

It is mandatory to ensure that sensitivity afforded by the identified terminal configuration is universally applicable to all types of windings (i.e., continuous-disc and interleaved-disc). Finally, authors believe that present findings would form valuable inputs for making a recommendation of the terminal connection most ideally suited for making frequency-response measurements in the relevant standards [6].

\section{LiterATURE REVIEW AND MOTIVATION}

Publications pertinent to the present study were gathered and analyzed. Many of them have discussed the importance of sensitivity, as well as, the influence of terminal connection on resonant frequencies. In the context of the present study, they can broadly be grouped into the following categories-

- Influence of terminal condition on natural frequencies [11]-[14]

Computational methods for determining steady-state voltage and current distribution in coils due to forced and free-oscillations, and their resonant behavior, was proposed. Further, incorporating the influence of terminal conditions, point of excitation and phase connections of single and three phase transformer windings on resonant frequencies was also reported. In the present context, it is important to note the observation made in [13], that in some terminal connections, a few natural frequencies are rendered nonexcitable. Obviously, such connections are best avoided.

\section{- Detection of winding deformation by SFRA}

Sensitivity achievable in detection of winding deformation, using network analyzers and sweep generators was reported, perhaps, for the first time, by Dick and Erven [15]. Another widely accepted publication,viz. the ERA Technology report [8] on frequency domain testing method, specifies a preferred terminal condition (nontested windings are to be kept open-circuited) and quantity to be measured. In the post digital-era, there have been many efforts to correlate winding deformation to changes in amplitude response of transfer function, for different terminal connections [16]-[19]. In these studies, some connections were observed to be more sensitive to a particular type of deformation. Hence, a question that naturally arises is, which connection is the best to use?

\section{- Terminal connections in LVI tests}

Recommendation on terminal connections and measurable quantities to be employed during LVI tests is mentioned in IEEE Standard [7]. Specifically, it states that both winding neutral ends are to be grounded and secondary winding line-end kept open. Contrastingly, in SFRA measurements, the existing industrial testing procedure is either to keep nontested windings open and/or short circuited with neutral floating. So, the connections used in these two tests are not the same. Naturally, the question that arises is which of them is more sensitive and what is the basis for this choice? These questions need to be answered.

\section{- IEEE Draft on SFRA measurements [6]}

During the review stages of this paper, this IEEE guide was made available to the authors by the referee. This is a draft currently under preparation by the IEEE Working Group for use as a guide for the application and interpretation of frequency response analysis for oil immersed transformers. It provides necessary guidelines for making FRA measurements, test procedures, test connections, test environment and test object preparations, suggestions on test leads and measurement types. An exhaustive list of various test configurations for different types of transformers has also been included. Although, some connections are currently being practiced during SFRA measurements, the draft does not recommend any particular terminal connection as preferred. Finally, some hints have also been provided for acquisition and interpretation of monitored data. From the literature review the following observations can be made.

1) FRA measurements have become universally accepted for detecting winding deformation.

2) Network/Impedance analyzers are now being extensively employed for this purpose.

3) In recent years, the practice has been to keep nontested windings as open and/or short circuited with neutral floating. 
Therefore, it emerges that a test configuration that exhibits maximum sensitivity (that which reveals more number of resonant peaks) has, so far, not been identified, and hence forms the subject matter of this paper.

\section{ADOPTED APPROACH}

To resolve these matters, initially a simple two-winding transformer was considered. Low voltage, sweep frequency measurement essentially comprises of applying a spectrally pure sinusoid to the transformer, followed by measurement of a suitable input and output quantities, at each discrete frequency. Once input and output quantity are decided, there is freedom to impose any valid condition on remaining terminals. Therefore, all possibilities arising from combinations of terminal connections, measurable quantities, and system functions will have to be included in this search exercise. It is easy to visualize that there exist several possibilities and the search-space is large. For clarity and ease of explanation, these possibilities have been grouped, based on status of line and neutral terminal of each winding. This is pictorially depicted in Fig. 1, along with measurable currents and/or voltages. The currently practiced terminal connections employed during SFRA measurements are indicated in Fig. 1. In all, 14 different configurations result. For a given network, a variety of system functions (such as driving-point, transfer, and gain) can be defined and estimated [20]. The possible options are indicated in Table I. For each configuration in Fig. 1, five measurable quantities were selected. Amongst them, two pertain to input (i.e. input voltage and current), while the remaining three are responses. Hence, for each configuration, six system functions (involving an input and response) can be computed. By definition, driving-point function and transfer function are ratios involving spectra of input quantity, and their poles correspond to natural frequencies of the network. Additionally, one can also define gain functions (or ratios), involving any two arbitrary quantities. The drawback with such ratios is, poles and zeros of such functions do not correspond to natural frequencies of the network. For example, when both neutrals are floating, a ratio of two neutral voltages can be computed, and similarly, when neutrals are grounded, ratio of two neutral currents can be considered. Peaks in the amplitude plots of these functions will not correspond to natural frequencies (for details, see Appendix). In fact, these frequencies cannot be related to natural frequencies of the network [21], [22]. So, in the present context of improving fault-detection sensitivity, considering such functions is of little use. Hence, care was exercised to avoid considering such functions. In addition to these possibilities, the method of connecting the source to the network is yet another factor to be accounted. Namely, the source could be connected between line and ground or between line and neutral. This matter has also been considered. Thus, in all there exist 14 configurations, six system functions, and two ways of excitation, implying $14 \times 6 \times 2=168$ different possibilities. During measurements it is common practice to transmit voltage/current signal via a co-axial cable to the measuring instrument, for purposes of noise/interference immunity. Under these conditions, the instrument could either be terminated by the surge impedance $(50 / 75 \Omega)$ or left open (1 M $\Omega$ impedance).
The mode of terminations employed can have significant influence, especially at higher frequencies. Therefore, both $(50 \Omega$, as well as $1 \mathrm{M} \Omega$ ) terminations were considered. This is in consonance with the currently employed practice of employing network/impedance analyzers, in which the measurements are always made with a $50 \Omega$ termination. In view of this factor, there will now exist $168 \times 2=336$ different possibilities which requires to be examined.

Initially, simulation studies were performed and later validated on an actual transformer. For this purpose, the well established coupled ladder network was employed, and is shown in Fig. 2. The circuit constants (Table II) were selected such that all natural frequencies are well below $1 \mathrm{MHz}$, so as to satisfy the upper frequency limitation of this quasi-static lumped-parameter circuit. This representation is known to adequately describe most aspects of impulse behavior of a transformer. Using PSPICE, all system functions were computed for each configuration shown in Fig. 1. Amplitude frequency responses of these functions were normalized (with respect to the highest peak) and the number of distinctly occurring peaks (i.e. natural frequencies) were counted. Depending on the number of peaks observable (peak $>0.05$ p.u., i.e., $5 \%$ ), each configuration was ranked. Subsequently, this ranking was verified for its invariance with respect to the type of winding. This was achieved as follows. For each configuration, the series capacitance of primary and secondary are varied to simulate different types of winding (i.e., lower series capacitance implies continuous-disc, while higher series capacitance represents interleaved winding).

\section{Simulation STUdiES}

The equivalent circuit (to represent a two-winding transformer) employed in the present study is shown in Fig. 2. The primary and secondary windings were represented with four sections each. It is well known that in such a circuit, the number of natural frequencies is equal to the total number of independent nodes [23]. For example, terminal connection "bfloat-oc" (see Fig. 1) when represented by a 4-section circuit, has nine independent nodes, and hence, will have nine natural frequencies, while connection "bfloat-sc" will have eight natural frequencies. Likewise, the number of natural frequencies for all other terminal connections were determined.

Using "AC Analysis" in PSPICE, amplitude frequency responses of system functions were computed, corresponding to every terminal configuration shown in Fig. 1. Additionally, analytical calculations were also performed to compute poles and zeros of each system function. After each simulation, the number of distinctly observable peaks in the amplitude response of the system function was counted manually. This exercise was repeated for different values of series capacitance in primary and secondary winding (i.e. for different values of “ $\alpha$ ", namely, 3, 4, 5, 6, 7, 8, and 9). For brevity, only a sample of these results is compiled in Table III. Most of the results in Table III correspond to measurements with $1 \mathrm{M} \Omega$ termination. In addition, only those results corresponding to the currently practiced terminal connections during SFRA measurements (with a $50 \Omega$ termination) are included and marked with $\mathrm{a} \star$. In each case, the number of natural frequencies actually identified 


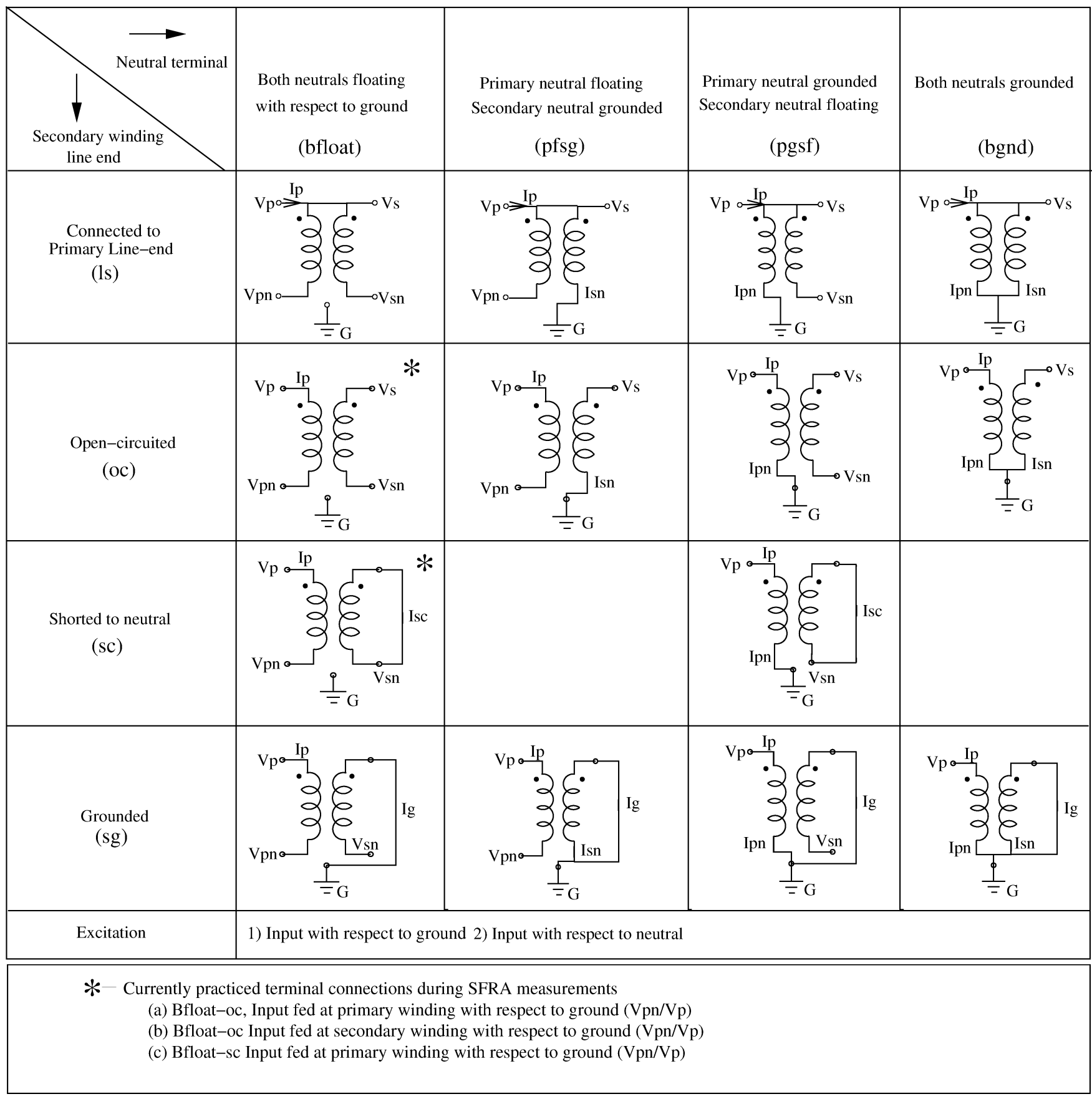

Fig. 1. Terminal configurations and measurable quantities.

TABLE I

SYSTEM FUNCTIONS

\begin{tabular}{l|c|c}
\hline \multicolumn{3}{c}{ System functions } \\
\hline Driving-point functions & \multicolumn{2}{|c}{ Transfer functions } \\
\hline $\begin{array}{c}\text { Driving-point impedance } \\
\text { and admittance }\end{array}$ & $\begin{array}{c}\text { Transfer impedance } \\
\text { and admittance }\end{array}$ & $\begin{array}{c}\text { Voltage* and } \\
\text { current gain }\end{array}$ \\
\hline $\begin{array}{l}*-\text { Currently practiced terminal connections during SFRA measurements } \\
\text { belong to this category }\end{array}$
\end{tabular}

and the theoretically expected maximum is indicated. This comparison provides an indication of the extent to which a particular pair of system function and configuration is good or bad. Further, for each configuration, the variance with respect to primary and secondary winding $\alpha^{\prime} s$ is evident from results presented in each row. When the number of natural frequencies identifiable is consistently high for all $\alpha^{\prime} s$, such a configuration can be rated as "good." Based on these criteria, all the $168 \times 2$ system functions were ranked and Table III shows a very small and important portion of this huge list. Each configuration is classified as "good," "reasonable," "medium," and "bad," accordingly. The amplitude frequency response plot of system function, corresponding to the "good" and "bad" configuration is shown in Fig. 3(a) and (b), respectively. While computing these responses, the incremental frequency was kept 


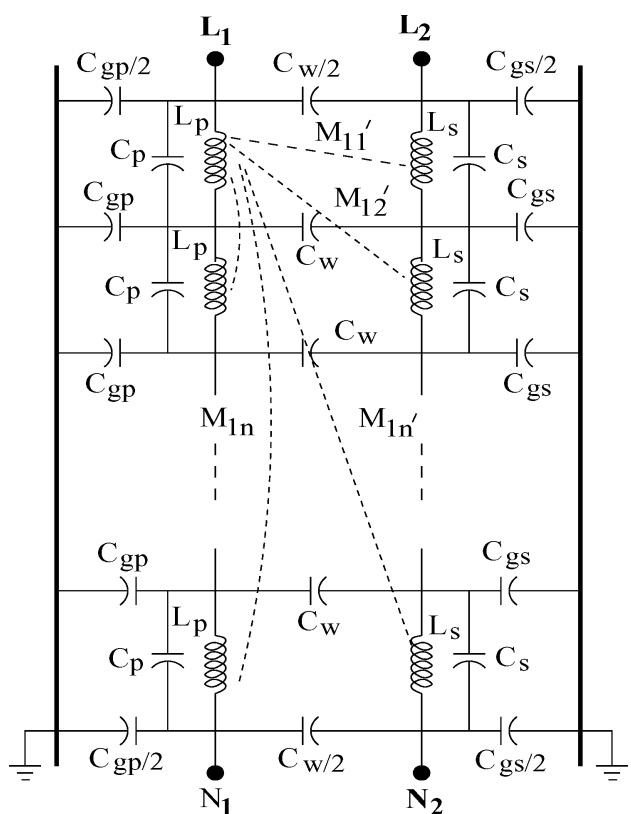

Fig. 2. Equivalent-circuit representation of a two-winding transformer.

TABLE II

CiRCUIT PARAMETERS

\begin{tabular}{|c|c|c|c|}
\hline \multicolumn{4}{|c|}{ Number of sections $(n=4)$} \\
\hline \multicolumn{4}{|c|}{$\begin{array}{c}\text { Primary winding resistance }\left(r_{p}=1.2 \Omega\right) \\
\text { Secondary winding resistance }\left(r_{s}=0.4 \Omega\right)\end{array}$} \\
\hline $\begin{array}{c}\text { Primary } \\
\text { winding } \\
\text { self and mutual } \\
\text { Inductance } \\
(\mathrm{mH}) \\
\end{array}$ & $\begin{array}{c}\text { Secondary } \\
\text { winding } \\
\text { self and mutual } \\
\text { Inductance } \\
(\mathrm{mH})\end{array}$ & $\begin{array}{c}\text { Mutual } \\
\text { inductance } \\
\text { between } \\
\text { windings } \\
(\mathrm{mH}) \\
\end{array}$ & Capacitances \\
\hline$L_{p}=0.180$ & $L_{s}=0.065$ & $M_{11}=0.0545$ & \multirow{4}{*}{$\begin{array}{l}C g_{\text {eff }}=6 n F \\
\text { Cs chosen so that } \\
\alpha \text { is } 3,4, \ldots 9\end{array}$} \\
\hline$M_{12}=0.1108$ & $M_{12}=0.036$ & $M_{12}=0.052$ & \\
\hline$M_{13}=0.074$ & $M_{13}=0.025$ & $M_{13}=0.042$ & \\
\hline$M_{14}=0.0547$ & $M_{14}=0.018$ & $M_{14}=0.033$ & \\
\hline
\end{tabular}

* Other mutual inductances are determined by symmetry

identical and equal to $500 \mathrm{~Hz}$. The distinctly identifiable peaks ( $>0.05$ p.u.) in each amplitude response is marked, and it can be seen that while the "good" connection revealed 7 natural frequencies (out of a maximum of 8), the "bad" one showed-up only 2 (against a maximum of 8 ). These results are compiled in Table III for different " $\alpha$ " values. The position occupied by the currently practiced terminal connections employed during SFRA measurements is highlighted in the Table III. Thus, influence of terminal connection on the observable natural frequencies is clear, and it implies that any arbitrary connection should not be employed. In addition, corresponding to these two extreme cases (i.e. "good" and "bad"), poles and zeros of the system function were determined and are plotted in Figs. 4 and 5, respectively. The pole-zero plot assists in demonstrating why each system function exhibits a different number of natural frequencies. As can be seen, in the "bad" case (Fig. 5), there are many near pole-zero cancellations, which explains why only two natural frequencies are observable. No such cancellations are observed in the "good" case (Fig. 4). By merely looking at the amplitude frequency response plot, such an explanation cannot be supported. So, importance of knowing both pole and zero is demonstrated [9]. In summary, from these simulations, the combination of terminal connection and system function that is ideally suited for frequency response measurements (i.e. yielding more number of natural frequencies) was identified. A schematic representation of the terminal connection which can be regarded as "good" along with quantities to be measured for computing transfer function is shown in Fig. 6. The process of ranking each configuration also led to the identification of some configurations that ought to be avoided. These results are next verified on an actual transformer. Prior to experiments on an actual transformer, the simulation results were initially validated by conducting experiments on a table-top model coil, and the results were found to be in good agreement. For brevity, these results are not reported.

\section{EXPERIMENTAL VALIDATION}

A $6.9 \mathrm{kV} / 11 \mathrm{kV}, 315 \mathrm{kVA}$, HV transformer (used for operating-duty-test of surge arresters) was selected. The experiments involved measuring the amplitude frequency response data corresponding to all the terminal configurations mentioned in Table III. For this purpose, an arbitrary waveform generator (producing $20 \mathrm{Vp}-\mathrm{p}$ sinusoid), a $150 \mathrm{MSa} / \mathrm{s}$, 8-bit digital oscilloscope, and a clamp-on current probe (sensitivity: $2 \mathrm{~mA} / \mathrm{mV}$, bandwidth: $450 \mathrm{~Hz}-60 \mathrm{MHz}$ ) were used. All the signals were connected to the instruments via a $50 \Omega$ co-axial cable having a maximum length of $1 \mathrm{~m}$. Suitable arrangements were made to connect cable to the transformer terminal. The transformer terminals were shorted and/or grounded using a flat $7 \mathrm{~cm}$ wide aluminum strip. Precautions were exercised to keep the length of these leads as short as practicable. The same set of connecting leads and cables were used throughout the experiments. The oscilloscope was either kept open circuited $(1 \mathrm{M} \Omega, 25 \mathrm{pF})$ or terminated using a $50 \Omega$ impedance, as the case may be. An automatic procedure was adopted for gathering the sweep frequency measurement data, and later transferred to a PC for plotting. Experimental results corresponding to both $1 \mathrm{M} \Omega$ and $50 \Omega$ terminations are presented in Table IV.

During practical measurements, counting of peaks in magnitude plot can become somewhat subjective. To circumvent this ambiguity the following procedure was used.

- An automatic sweep frequency measurement was done and normalized magnitude plots obtained. All dominant peaks are counted and labeled.

- In the magnitude plot, frequency intervals pertaining to peak-like kinks are noted, and for this frequency interval, a manual sweep frequency measurement is done. During which, vertical scale of oscilloscope is dynamically changed each time to ensure full-scale-deflection (This feature could not be incorporated in the automated measurement procedure).

- Additionally, to make identification of peaks foolproof, occurrence of zero-crossing of phase angle (i.e., angle between input and output) was ensured. Thus, it was guaranteed that all peaks counted and labeled (however small in magnitude), was indeed a pole. 
TABLE III

NUMBER OF PEAKS

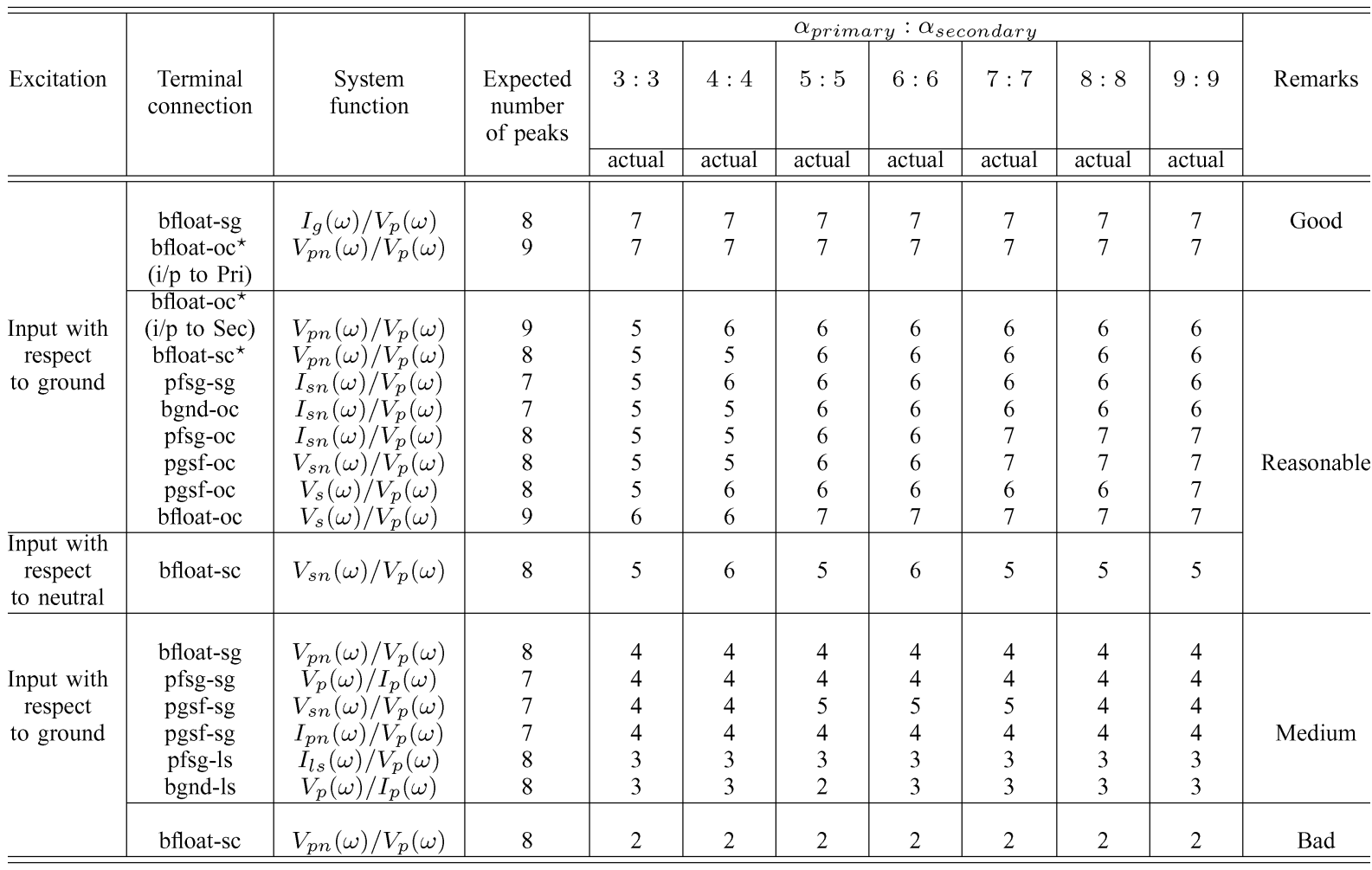

* - Currently practiced terminal connections during SFRA measurements (with $50 \Omega$ termination)

- All the remaining data pertain to measurements made with $1 \mathrm{M} \Omega$ termination
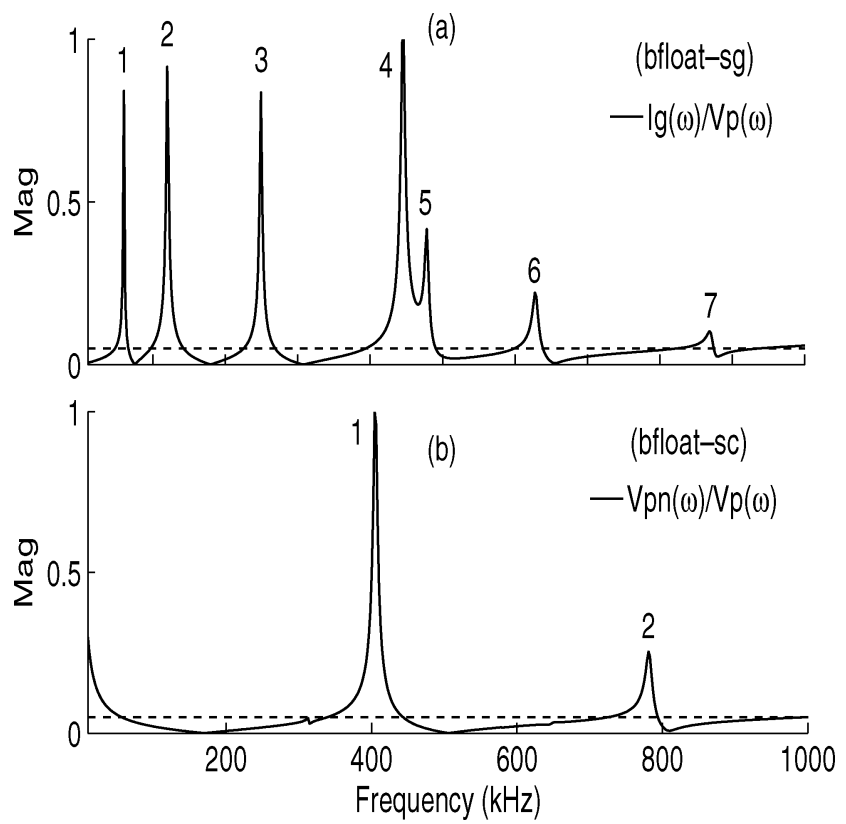

Fig. 3. Amplitude frequency response of system function (with $1 \mathrm{M} \Omega$ termination) (a) yielding more peaks and (b) yielding fewer peaks.

Fig. 7(a) shows amplitude frequency response corresponding to the "good" configuration in Table IV and pertaining to $50 \Omega$ termination. In this figure, 14 natural frequencies are observable, and each of them has been numbered. Next, consider, Fig. 7(c), which corresponds to the "bad" configuration

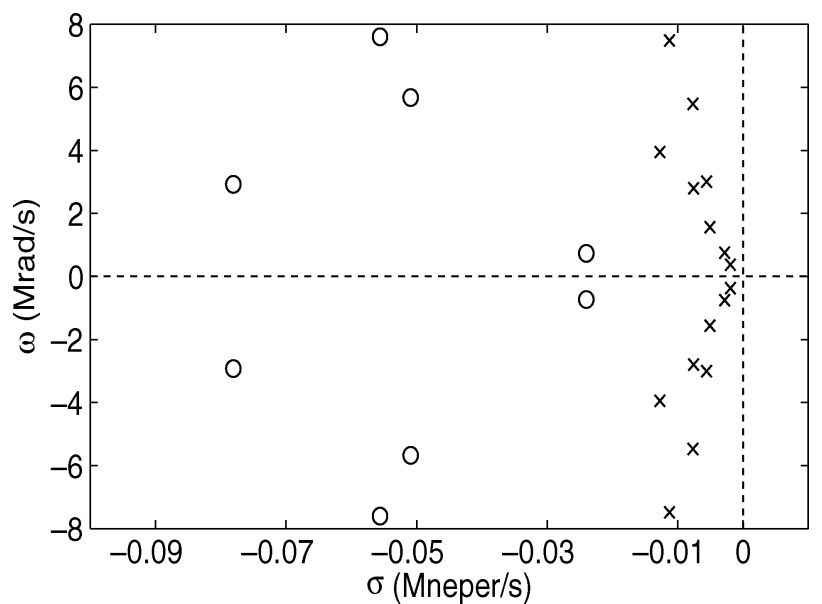

Fig. 4. Pole-zero plot of system function corresponding to Fig. 3(a).

in Table IV, and only 4-5 natural frequencies are observable. Comparing these two figures, it becomes evident that by choosing an appropriate pair of terminal connection and system function, the achievable sensitivity can be significantly improved. Incidentally, these two figures are adequate evidence about the importance of the objective of this paper. Another example is shown in Fig. 7(b), which belongs to the "medium" category in Table IV, yielding about 8-10 natural frequencies.

As can be seen from Table IV, the number of peaks which can be detected/observed from the amplitude response plot, for both $1 \mathrm{M} \Omega$ and $50 \Omega$ termination conditions, is nearly the same. 


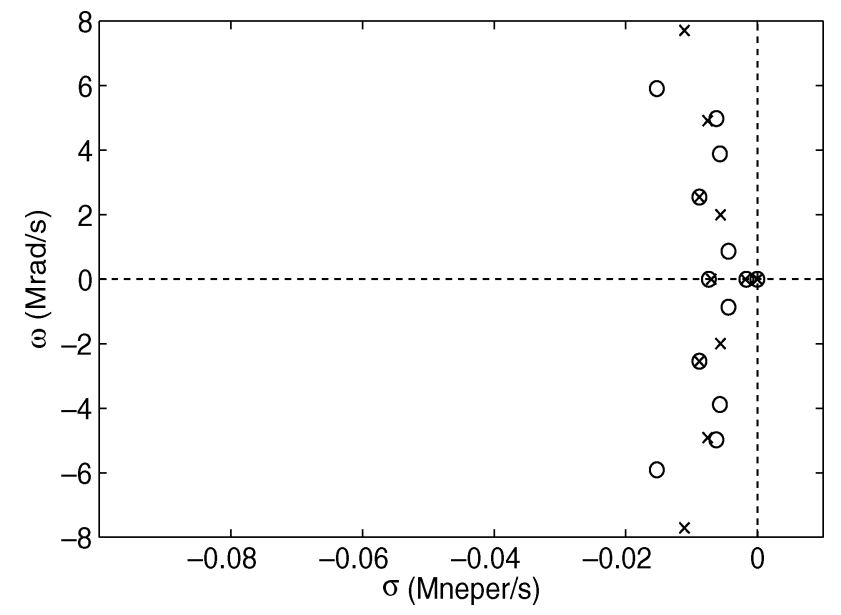

Fig. 5. Pole-zero plot of system function corresponding to Fig. 3(b).

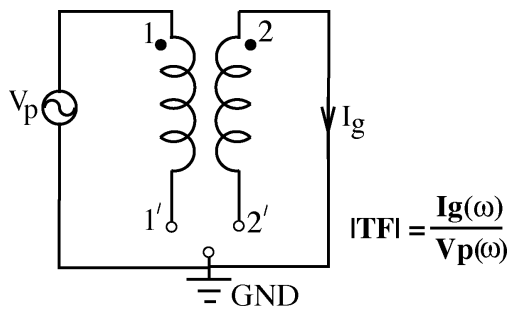

Fig. 6. Schematic representation of configuration and its system function identified to be "good".

TABLE IV

EXPERIMENT ON AN OPERATING-DUTY TESTING TRANSFORMER-TERMINAL CONFIGURATIONS AND NUMBER OF PEAKS

\begin{tabular}{|c|c|c|c|c|c|}
\hline \multirow[t]{2}{*}{$\begin{array}{c}\text { Excitation } \\
\text { Method }\end{array}$} & \multirow[t]{2}{*}{$\begin{array}{c}\text { Terminal } \\
\text { Connection }\end{array}$} & \multirow[t]{2}{*}{$\begin{array}{c}\text { System } \\
\text { Function }\end{array}$} & \multicolumn{2}{|c|}{$\begin{array}{l}\text { Number } \\
\text { of peaks }\end{array}$} & \multirow[t]{2}{*}{ Remark } \\
\hline & & & $1 M \Omega$ & $50 \Omega$ & \\
\hline \multirow[b]{2}{*}{$\begin{array}{l}\text { Input } \\
\text { w.r.t } \\
\text { ground }\end{array}$} & $\begin{array}{c}\text { bfloat-sg } \\
\text { bgnd-oc }^{\dagger} \\
\text { pfsg-oc } \\
\text { bfloat-oc* }\end{array}$ & $\begin{array}{c}I_{g}(\omega) / V_{p}(\omega) \\
I_{s n}(\omega) / V_{p}(\omega) \\
I_{s n}(\omega) / V_{p}(\omega) \\
\mathrm{V}_{p n}(\omega) / V_{p}(\omega)\end{array}$ & $\begin{array}{c}14 \\
12 \\
12 \\
7 \\
\end{array}$ & $\begin{array}{l}14 \\
12 \\
12 \\
12 \\
\end{array}$ & Good \\
\hline & $\begin{array}{c}\text { pfsg-sg } \\
\text { pfsg-sg } \\
\text { pgsf-sg } \\
\text { pgsf-oc } \\
\text { bfloat-sg } \\
\text { pgsf-oc } \\
\text { bfloat-oc* } \\
\text { (Input at } \\
\text { secondary) } \\
\text { bfloat-sc* }\end{array}$ & $\begin{array}{c}I_{s n}(\omega) / V_{p}(\omega) \\
V_{p}(\omega) / I_{p}(\omega) \\
I_{p n}(\omega) / V_{p}(\omega) \\
V_{s n}(\omega) / V_{p}(\omega) \\
V_{p n}(\omega) / V_{p}(\omega) \\
V_{s}(\omega) / V_{p}(\omega) \\
V_{p n}(\omega) / V_{p}(\omega) \\
V_{p n}(\omega) / V_{p}(\omega)\end{array}$ & $\begin{array}{l}10 \\
10 \\
10 \\
9 \\
9 \\
7 \\
\\
7 \\
\\
4\end{array}$ & $\begin{array}{l}10 \\
10 \\
10 \\
10 \\
10 \\
10 \\
\\
7 \\
\\
9\end{array}$ & Medium \\
\hline $\begin{array}{c}\text { Input } \\
\text { w.r.t } \\
\text { neutral }\end{array}$ & bfloat-sc & $V_{p n}(\omega) / V_{p}(\omega)$ & 7 & 7 & \multirow[t]{2}{*}{ Fair } \\
\hline Input & bgnd-ls & $V_{p}(\omega) / I_{p}(\omega)$ & 7 & 7 & \\
\hline ground & pfsg-ls & $I_{l s}(\omega) / V_{p}(\omega)$ & 5 & 5 & $\mathrm{Bad}$ \\
\hline
\end{tabular}

*-Currently practiced during SFRA measurements

$\dagger$-Recommended by IEEE standards during LVI testing

However, an inspection of the amplitude response plots for both terminations clearly reveals some important differences. The peak value of the natural frequencies in the plots is significantly
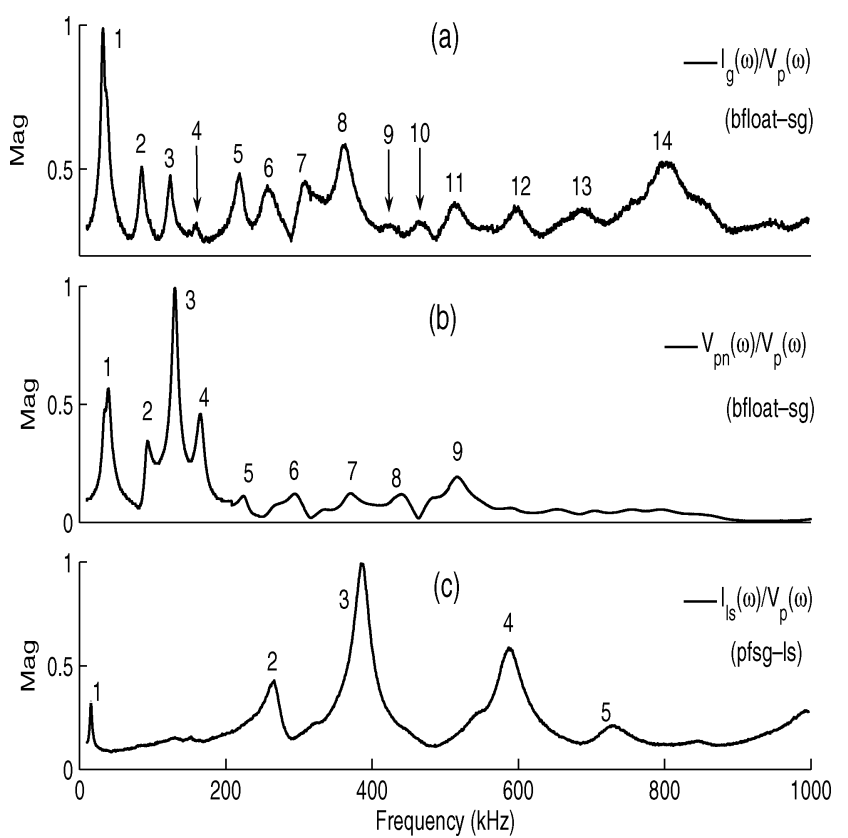

Fig. 7. Measured amplitude frequency response pertinent to configuration: (a) good, (b) medium, and (c) bad, measured with $1 \mathrm{M} \Omega$ termination.

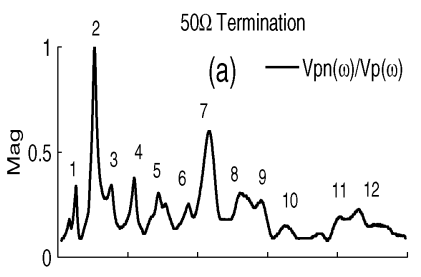

${ }_{5}$ (b) $-\operatorname{Vpn}(0) / \mathrm{Np}(0)$
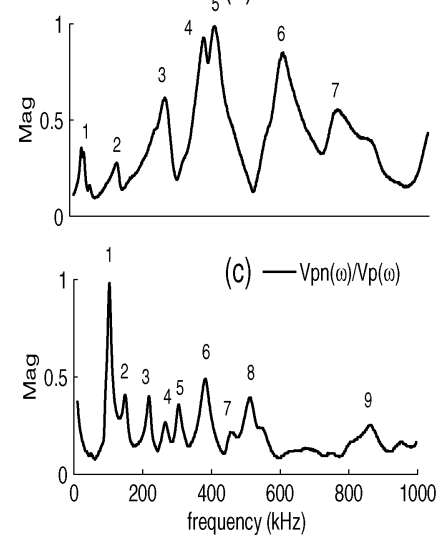

$1 \mathrm{M} \Omega$ Termination

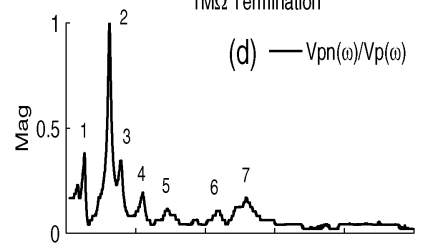

(e) $-V \operatorname{vp}(\omega) / N p(\omega)$
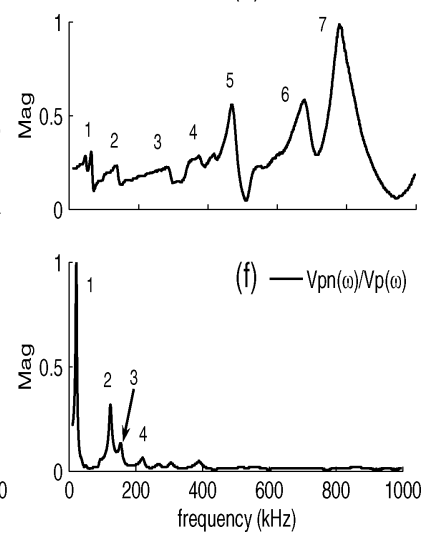

Fig. 8. Currently practiced terminal connections during SFRA measurements-(a), (b), (c) with $50 \Omega$ termination and (d), (e), (f) with $1 \mathrm{M} \Omega$ termination (subplot sequence is same as that in Table IV).

higher (beyond about $400 \mathrm{kHz}$ ) for the $50 \Omega$ termination compared to the $1 \mathrm{M} \Omega$ termination. This aspect is illustrated in Fig. 8 corresponding to the currently practiced terminal connections during SFRA measurements.

While on this point, it is worth mentioning that the system function involves a ratio of two voltages, the method of termination used becomes significant (Fig. 8). However, when one of the two quantities in the ratio is a current, then both terminations yield very similar results (this aspect is seen in Table IV). From this it is evident that, measurements carried out with a 50 

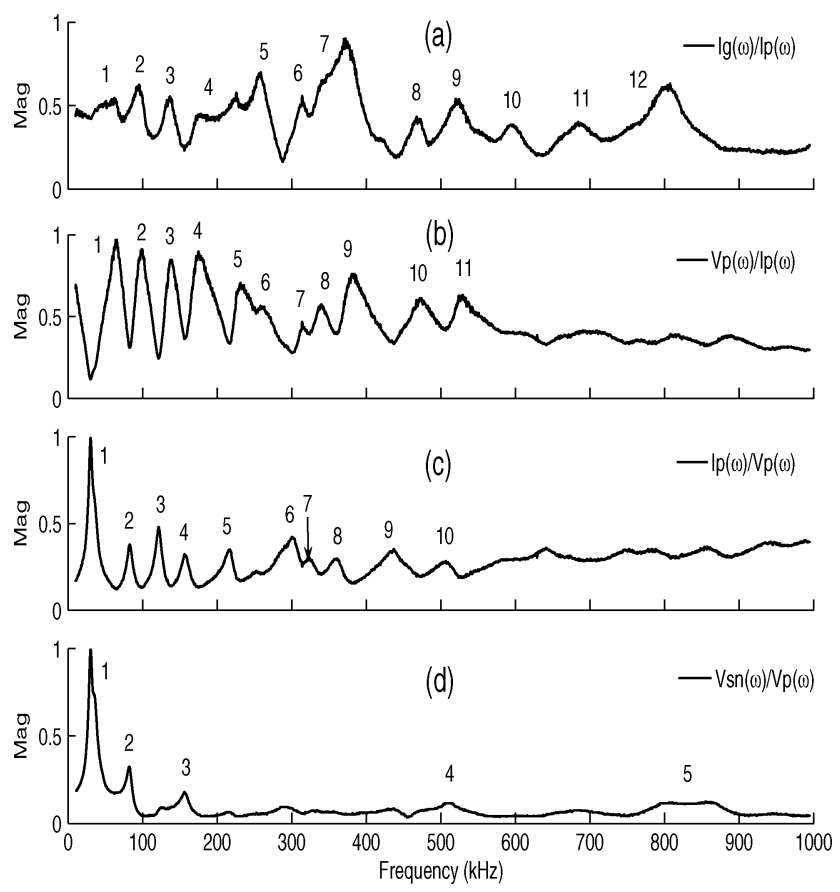

Fig. 9. Measured amplitude frequency response corresponding to the other system functions while adopting the "good" configuration (bfloat-sg) pertaining to $1 \mathrm{M} \Omega$ termination.

$\Omega$ termination are superior compared to the $1 \mathrm{M} \Omega$ termination. It is very easy to visualize that this superiority will be much more pronounced when measurements are carried out on large power transformers (on-site), which invariably involve longer cable lengths, increased noise levels, interference etc. Thus, the $50 \Omega$ terminated measurements are preferable.

Some important portions of the experimental results (corresponding to actual transformer) are compiled in Table IV. Although, the actual ranking of configurations arising out of experimental results do slightly differ from those obtained from simulation (which was naturally expected), the overall sequence remains more or less unaltered, viz. the position occupied by the "good" and "bad" configurations has not been disturbed. In other words, the "good" and "bad" configurations identified in simulation studies are observed to be true even during measurements on actual transformers. Thus, an indirect validation of simulation results was achieved.

Employing the "good" terminal configuration, other possible system functions were considered, and the result of these measurements ( $1 \mathrm{M} \Omega$ termination) are depicted in Fig. 9. In each case, the system function considered and the number of frequencies observable are indicated. It can be seen that, in all these cases, only about 5-12 peaks are observable.

So, it emerges that merely identifying a terminal connection is alone not sufficient, but, an optimal combination of terminal connection and system function has to be found. The task of finding this combination involved a vast search-space. In this work, a brute-force search approach was employed with the constraint that the number of natural frequencies observable should be a maxima. Extensive field measurements on different transformers will assist in reconfirming universality of the identified configuration.

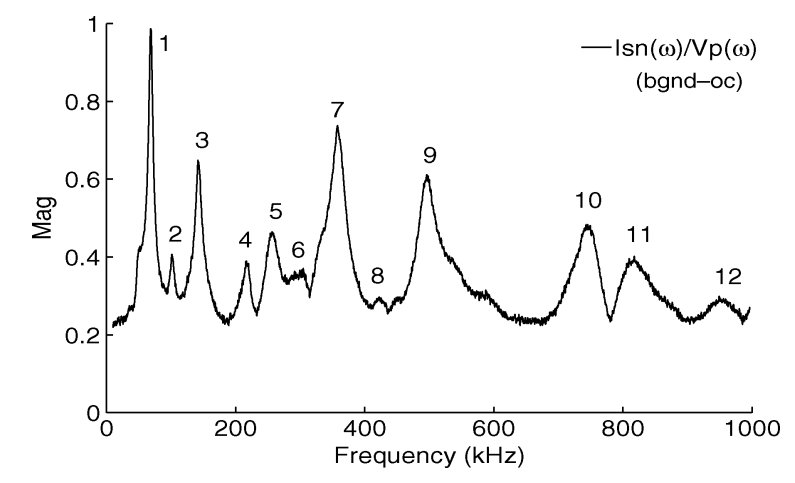

Fig. 10. Measured amplitude frequency response by adopting LVI test connection (1 M $\Omega$ termination).

Next, the terminal connection recommended by IEEE standard [7] for use during LVI testing was also specifically implemented, in order to ascertain its position with respect to the identified configuration. Experiments were conducted imposing the LVI test connections. The results are presented in Fig. 10 (for a $1 \mathrm{M} \Omega$ termination). Compared to 14 peaks identified from the "good" configuration, the LVI test connection yielded about 11-12 peaks, a marginal decrease. Arising out of these experiments, a rank list of various configurations was made, and the more important portions of this is compiled in Table IV. The LVI test connection occupies a position adjacent to the "good" connection. In comparison to simulation results, it is seen that some configurations occupy slightly different ranks.

As discussed earlier, experiments were also specifically conducted by imposing connections currently being practised during SFRA measurements employing both types of terminations. The results were presented in Fig. 8 and the position occupied by these connections is included in Table IV. It is observed that one amongst the currently practiced terminal connections is almost as good as the identified "good" configuration (see Table IV). Thus, by adopting the identified configuration, improvement in sensitivity during SFRA measurements can be expected.

\section{CONCLUSION}

A systematic study comprising of simulation, analytical computation and experiments on an actual transformer were carried out to find the most appropriate combination of terminal connection and system function, which would be ideal for making frequency response measurements on transformers. Finding the ideal configuration was based on an exhaustive search of possible combinations, eventually leading to identification of one pair of terminal-connection and system-function, from which the maximum number of natural frequencies could be observable. Experiments on an actual transformer validated the results, i.e. proved that the identified configuration was superior compared to the rest of them. This study demonstrated that a significant improvement in sensitivity (i.e., an increased number of natural frequencies is observable) can be achieved by merely employing a different terminal configuration during frequency response measurements. Further, it also provided a quantitative 
assessment of sensitivities achievable from other possible configurations as well. In this context, the contribution is novel, original and of practical relevance.

Future work ought to focus on evaluating efficacy of the proposed configuration by extensive experiments on large 3-phase and multiwinding transformers. A more realisitic picture about the efficacy of various connections would emerge when extensive measurements on actual transformers are done. It is hoped, that such an exercise would attract active support and involvement of power utilities. Once its suitability is adequately confirmed, it would serve as a valuable input for inclusion into the relevant standards (currently under preparation) on low-voltage sweep frequency-response measurements on transformers.

\section{APPENDIX}

For example, consider a configuration in which, both neutrals are floating and let $V_{p n}, V_{s n}$ be the primary and secondary neutral voltages. Let $V_{p}$ be the input voltage. The neutral voltages are one of the responses of the system. So, for the primary neutral terminal, a system function can be defined as

$$
H_{1}(s)=\frac{V_{p n}(s)}{V_{p r i}(s)}=\frac{P(s)}{Q(s)}
$$

where roots of $Q(s)$ are the natural frequencies of the system, while roots of $P(s)$ the zeros.

In a similar fashion, for the secondary neutral terminal

$$
H_{2}(s)=\frac{V_{s n}(s)}{V_{p r i}(s)}=\frac{P^{\prime}(s)}{Q(s)}
$$

In both of the above cases, the denominator [i.e., Q(s)] remains the same, while the numerator (i.e., zeros) is different.

Now consider a system gain (i.e., a ratio in which two responses are involved), namely

$$
\begin{aligned}
H(s) & =\frac{V_{p n}(s)}{V_{s n}(s)} \\
& =\frac{V_{p n}(s) / V_{p r i}(s)}{V_{s n}(s) / V_{p r i}(s)}=\frac{P(s)}{P^{\prime}(s) .}
\end{aligned}
$$

It is seen that the numerator and denominator polynomials are zeros of (1) and (3), and in no way are related to natural frequencies of the system. Hence, such functions are to be excluded. This can be easily done by ensuring that one quantity in the ratio must necessarily correspond to the input (current or voltage).

\section{REFERENCES}

[1] IEC Standard Publication 722, Guide to the Lightning Impulse and Switching Impulse Testing of Power Transformer Reactors1st ed. 1982.

[2] IEEE Guide for Transformer Impulse Tests, IEEE Std. 93-1968, 1968.

[3] S. Tenbohlen and S. A. Ryder, "Making frequency response analysis measurements: A comparison of swept frequency and LVI methods," in Proc. XIIIth Int. Symp. High Voltage Engineering, The Netherlands, 2003, pp. 1-4.
[4] S. M. Islam, "Detection of shorted turns and winding movements in large power transformer using frequency response analysis," in IEEE Power Engineering Society Winter Meeting, Jan. 2000, pp. 2233-2238.

[5] M. Wang and A. J. Vandermaar, "Offline/online FRA condition monitoring for power transformer," IEEE Power Eng. Rev., pp. 54-56, Aug. 1999.

[6] IEEE PC57.149/D1, Draft Trial Use Guide for the Application and Interpretation of Frequency Response Analysis for Oil Immersed Transformers Mar. 2006.

[7] IEEE Guide for Short-Circuit Testing of Distribution and Power Transformers: Part-II, IEEE Std C57.12-90-1993, 1993, pp. 89-90.

[8] M. Bradson and D. G. Watterson, "The determination of winding displacement in power transformers by the use of static frequency domain measurement techniques-Theory and Method," ERA Report 96-0605(Issue2), Sep. 1996.

[9] L. Satish and A. Jain, "Structure of transfer function of transformers with special reference to interleaved windings," IEEE Trans. Power Del., vol. 17, no. 3, pp. 754-760, Jul. 2002.

[10] R. Malewski, R. C. Degeneff, and E. Troyan, "An assessment of transformer insulation dielectric strength under fast $\mathrm{HV}$ transients generated by SF6 breakers based on measurement of the winding transfer function," in CIGRÉ Session 2002.

[11] P. A. Abetti, "Survey and classification of published data on the surge performance of transformers and rotating machines," AIEE Trans., vol. 20, no. 5, pp. 1403-1414, Feb. 1959.

[12] S. Jayaram, "Influence of secondary winding terminal conditions on impulse distribution in transformer windings," Elect. Mach. and Power Syst., vol. 21, no. 3, pp. 183-198, 1993.

[13] B. I. Gururaj, "Influence of phase connections and terminal conditions on natural frequencies of three-phase transformer windings," IEEE Trans. Power App. Syst., vol. 87, no. 3, pp. 1-12, Jan. 1968.

[14] K. H. Sheshkamal, "Natural frequencies and transient responses of three-phase transformer windings," Elect. Mach. and Power Syst., vol. 15, no. 3, pp. 183-198, 1988.

[15] E. P. Dick and C. C. Erven, "Transformer diagnostic testing by frequency response analysis," IEEE Trans. Power App. Syst., vol. PAS-97, no. 6, pp. 2144-2153, Nov./Dec. 1978.

[16] M. Wang and A. J. Vandermaar, "Transformer winding movement monitoring in service-key factors affecting FRA measurements," IEEE Electr. Insul. Mag., vol. 20, no. 5, pp. 5-12, Sep./Oct. 2004.

[17] M. Wang and A. J. Vandermaar, "Improved detection of power transformer winding movement by extending the FRA high frequency range," IEEE Trans. Power Del., vol. 20, no. 3, pp. 1930-1938, Jul. 2005.

[18] E. Rahimpour, J. Christian, K. Feser, and H. Mohesin, "Transfer function method to diagnose axial displacement and radial deformation of transformer windings," IEEE Trans. Power Del., vol. 18, no. 2, pp. 493-505, Apr. 2003.

[19] M. Florkowski and J. Furgal, "Detection of transformer winding deformations based on the transfer function-measurements and simulations," Measur. Sci. and Technol., vol. 14, pp. 1986-1992, 2003.

[20] E. Brenner and M. Javid, Analysis of Electric Circuits, 2nd ed. New York: McGraw-Hill , 1967.

[21] E. A. Guillemin, Introductory Circuit Theory. New York: Wiley, 1963.

[22] E. A. Guillemin, Theory of Linear Physical Systems. New York: Wiley, 1963.

[23] T. S. Huang and R. R. Parker, Network Theory-An Introductory Course, 2nd ed. Reading, MA: Addison-Wesley, 1971.

L. Satish (SM'64) received the Ph.D. degree from the Indian Institute of Science (IISc), Bangalore, India, in 1993.

He was a post-doctoral fellow at ETH Zurich, Zurich, Switzerland, during 1993-1995. He joined the Department of High Voltage Engineering, IISc, in 1995 and is currently a Professor in the High Voltage Laboratory, Department of Electrical Engineering. His areas of research include application of signal processing to high-voltage impulse testing, diagnostics, and condition monitoring, and ADC testing. He was a Visiting Researcher at the High Voltage Institute, H.U.T, Finland, during the summer of 1998.

Dr. Satish is a member of CIGRE WG D1-33.

A. Saravanakumar (S'78) is a currently pursuing the Ph.D. degree in the High Voltage Laboratory, Department of Electrical Engineering, Indian Institute of Science, Bangalore, India. 